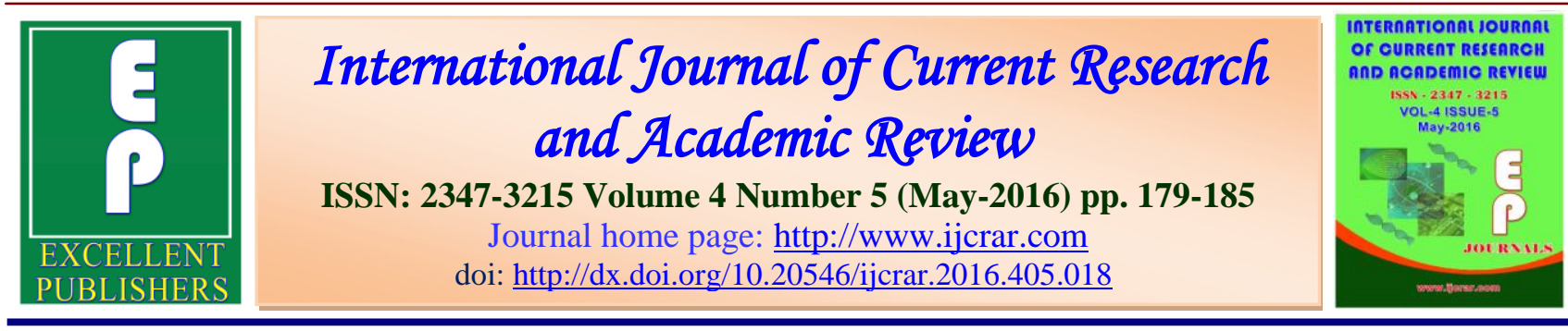

\title{
Evaluation of Interleukin IL-17A, IL-33, and Serum Levels of Zinc, Iron and Adenosine Deaminase in Cutaneous Leishmaniasis in Kirkuk, Iraq
}

\author{
Husain F. Hassan*, Sahla Kh. Abbas and Dunya S. Shakoor \\ Department of Biology, College of Science, University of Kirkuk, Iraq \\ *Corresponding author
}

\begin{tabular}{l|l}
\hline KEYWORDS & A B S T R A C T \\
$\begin{array}{l}\text { Interleukin, } \\
\text { zinc, iron, } \\
\text { adenosine } \\
\text { deaminase, } \\
\begin{array}{l}\text { cutaneous } \\
\text { leishmaniasis. }\end{array}\end{array}$ & $\begin{array}{l}\text { Serum levels of IL-17A, IL-33, zinc, iron and adenosine deaminase } \\
\text { were assessed in patients with cutaneous leishmaniasis. IL-17A, IL-33 } \\
\text { and adenosine deaminase were significantly increased in patients } \\
\text { compared to healthy subjects. In contrast zinc and iron were decreased } \\
\text { in sera of patients. These results suggest a role of IL-17A and IL-33 in } \\
\text { the pathogenesis of cutaneous leishmaniasis. }\end{array}$ \\
\hline
\end{tabular}

\section{Introduction}

Protozoan parasites of the genus Leishmania cause a spectrum of clinical disease, including cutaneous (CL), mucocutaneous (MCL) and visceral leishmaniasis (VL) (Sharma et al., 2008; Rahi et al., 2014). Approximately 12 million people are infected with this parasite worldwide with an incidence of 1.5-2 million new cases occurring each year (Garnier and Croft, 2002). All Leishmania parasites are dimorphic organisms which exist as extracellular promastigotes in the sandfly midgut and as intracellular amastigotes that lives in the phagolysosomes of macrophages in the mammalian host cells (Rahi, 3013). These diseases are transmitted by the bite of the infected female phlebotomine sandfly in the old world and Lutzomyia in the new world (Al-Warid, 2007).
In leishmaniasis two functionally distinct $\mathrm{T}$ helper (Th) cell subsets, Th1 and Th2, will decide whether there will be cure or progression of the disease. The action of Th1 (IFN- $-\gamma$, IL-2, TNF- $\alpha$ ) results in cure while action of Th2 cytokines (IL-4, IL-5, IL-10) results in progression of the disease. In cutaneous leishmaniasis, Th1 cytokines (IL-2 and $\mathrm{IFN}_{-}$) predominate over $\mathrm{Th} 2$ cytokines (IL-4) while in visceral and mucocutaneous leishmaniasis, there is increase in Th2 cytokines (Awasthi et al., 2004; Mutiso et al., 2013). Other Th17 cytokines like IL-17A has been reported to play a role in the pathogenesis of the inflammatory reaction in leishmaniasis (Bacellar et al., 2009; Olivia et al., 2009). On the other hand a newly described Th1 
cytokine (IL-33) has been suggested to be a new factor of susceptibility and a potential prognostic marker during visceral leishmaniasis (Rostan et al., 2013).

Here we described the role of IL-17A and IL-33 in the pathogenesis of cutaneous leishmaniasis. In addition we investigated whether the serum levels of zinc and iron as well as adenosine deaminase change in leishmaniasis patients.

\section{Materials and methods}

Patients: The sera from patients $(n=54)$ and healthy donors (17) were collected for IL17A, and IL-33 dosage.

\section{Assessment of IL-17A and IL-33 serum levels}

The assessment was carried out by using two ELISA kits, IL-17A (Cedarlane -US) and IL-33 (Bioassay Technology, Chinese), in which an anti- human IL-17A or IL-33 (Shanghai, China) coating antibody was adsorbed on to wells of 96 well plate. Human cytokine present in the standard or sample binds to antibodies that were adsorbed to the wells. A biotinylated anti human IL-17A or IL-33 antibody was then added and binds to human cytokine captured by the first antibody. Following incubation, unbound biotinylated anti- human cytokine antibody was removed during a wash step and avidin horse radish peroxidase (HRP) conjugate was then added, and binds to the biotinylated anti- human cytokine antibody. Following incubation unbound avidin- HRP conjugate was removed during awash step, and a substrate solution reactive with HRP was added to the wells. A colored product was formed in proportion to the amount of human cytokine present in the sample or standard. The color product was monitored with ELISA plate Biotek reader and absorbance was measured at a wave length of $405 \mathrm{~nm}$. A standard curve was plotted from standard of IL-17A and IL-33 and their level was determined from a curve fitting equation (Figure 1 and 2).

\section{Evaluation of $\mathrm{Zn}$ and $\mathrm{Fe}$ in $\mathrm{CL}$ patient serum}

Analysis of zinc and iron were measured by using flame atomic absorption spectrometry (Varian AA24OFS) according to the method of Kirbright (1980). Serum samples were diluted with deionized water. Different concentrations of trace elements were prepared for calibration of standard curves. Absorbances were read at $213.8 \mathrm{~nm}$ and $248.2 \mathrm{~nm}$ for zinc and iron, respectively.

\section{Assay of adenosine deaminase in CL patient serum}

The activity of adenosine deaminase was assayed in the serum of CL patients according to the procedure of Giusti, 1980, at a wavelength $620 \mathrm{~nm}$.

\section{Statistical analysis}

Statistical analysis to be performed using the Statistical Package for the Social Science (SPSS), a t-test was used to measure the difference in means between two groups. A value $\mathrm{P} \leq 0.05$ was considered statistically significant.

\section{Results and Discussion}

The comparative analysis between the patients with cutaneous leishmaniasis and non infected controls revealed significant increase for IL-17A and IL-33 (Table 1). IL33 also showed significant increased level in

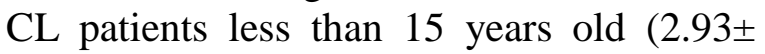
$0.31 \mathrm{pg} / \mathrm{ml}$ ) compared to CL patients more than 15 years old $(1.87 \pm 0.03 \mathrm{pg} / \mathrm{ml})$ (Table 2 ). 
On the other hand, IL-17A showed nonsignificant increased level in CL patients less than 15 years old $(40.57 \pm 4.58 \mathrm{pg} / \mathrm{ml})$ compared to CL patients more than 15 years old $(28.5 \pm 3.36 \mathrm{pg} / \mathrm{ml})$ (Table 2).

As presented in Table (3), the results also showed significant increased level for IL$17 \mathrm{~A}$ in female groups of patients as compared to male groups patients $(\mathrm{p} \leq 0.01)$, While there was numerical increased serum levels for il-33 in female patients compared to male patients.

In Table (4) and Table (5) the values of zinc and iron in the patients suffering with the cutaneous leishmaniasis were compared to the control group. A significant decrease in zinc level was observed between patients and healthy subjects $(\mathrm{p} \leq 0.01)$. Zinc level was found to be $(5.90 \pm 0.14 \mu \mathrm{mole} / \mathrm{lit})$ in patients, and this value was found to be statistically lower as compared with the controls $(8.74 \pm 0.03 \mu$ mole / lit $)$.

Mean while, no significant differences in status of iron was observed between the patients $(14.22 \pm 0.36 \mu$ mole. lit $)$ and control group $(22.12 \pm 0.73 \mu$ mole / lit). In contrast, the activity of adenosine deaminase was significantly higher in patients with cutaneous leishmaniasis than the control group (Table 6).
Cutaneous leishmaniasis is characterized by a wide spectrum of clinical manifestations ranging from the mild erythematous nodule (localized cutaneous leishmaniasis) to multiple non ulcerative nodules (diffuse cutaneous leishmaniasis) and the disfiguring mucosal form (mucocutaneous leishmaniasis) (Gonzalez et al., 2009). As a result of the interaction between different species of Leishmania, the vector and the mechanism of the immune response of the mammalian host, a spectrum of clinical, histopathological and immunopathological manifestations is observed in human (Carter et al., 2009; Liu and Uzonna, 2012). The types of immune response mounted by the individual, crucial for eliminating the parasites, drive such outcomes.

Such response, however, is dependent upon host genetic factors and the species of Leishmania responsible for infection (Sehgal et al., 2012). Factors associated with the immune response include tumor necrosis factor (TNF); an effective response of natural killers (NK) cells against interleukin -12 (IL-12); and an appropriate production of interferon gamma (IFN- $\gamma$ ) among other cytokines that might be regulated by the Leishmania responsible for infection (Awasthi et al., 2004; Sharma and Singh, 2009).

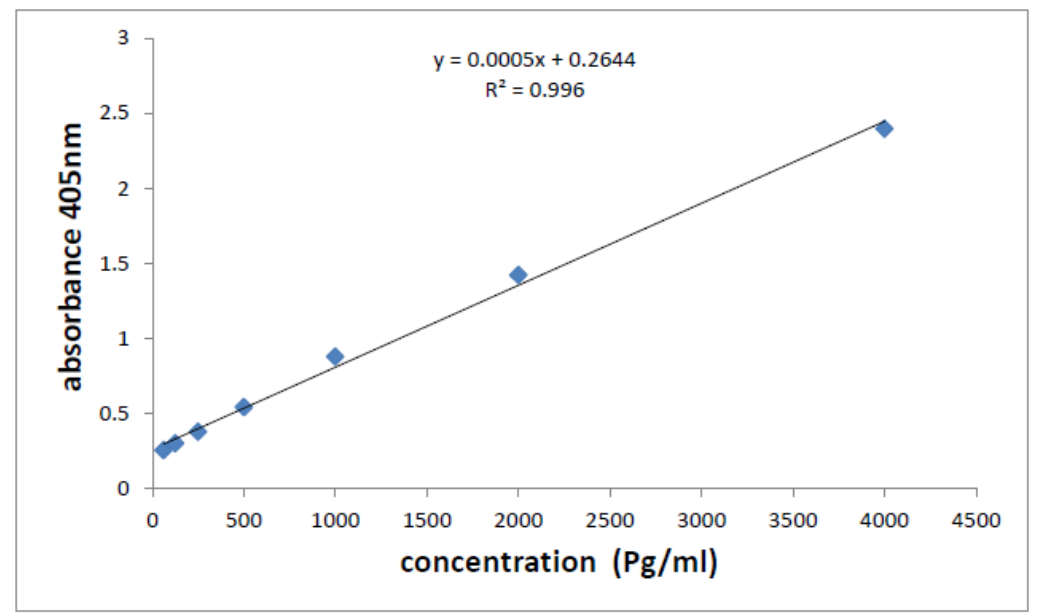

Figure.1 Standard curve of IL -17A 
Int.J.Curr.Res.Aca.Rev.2016; 4(5): 179-185

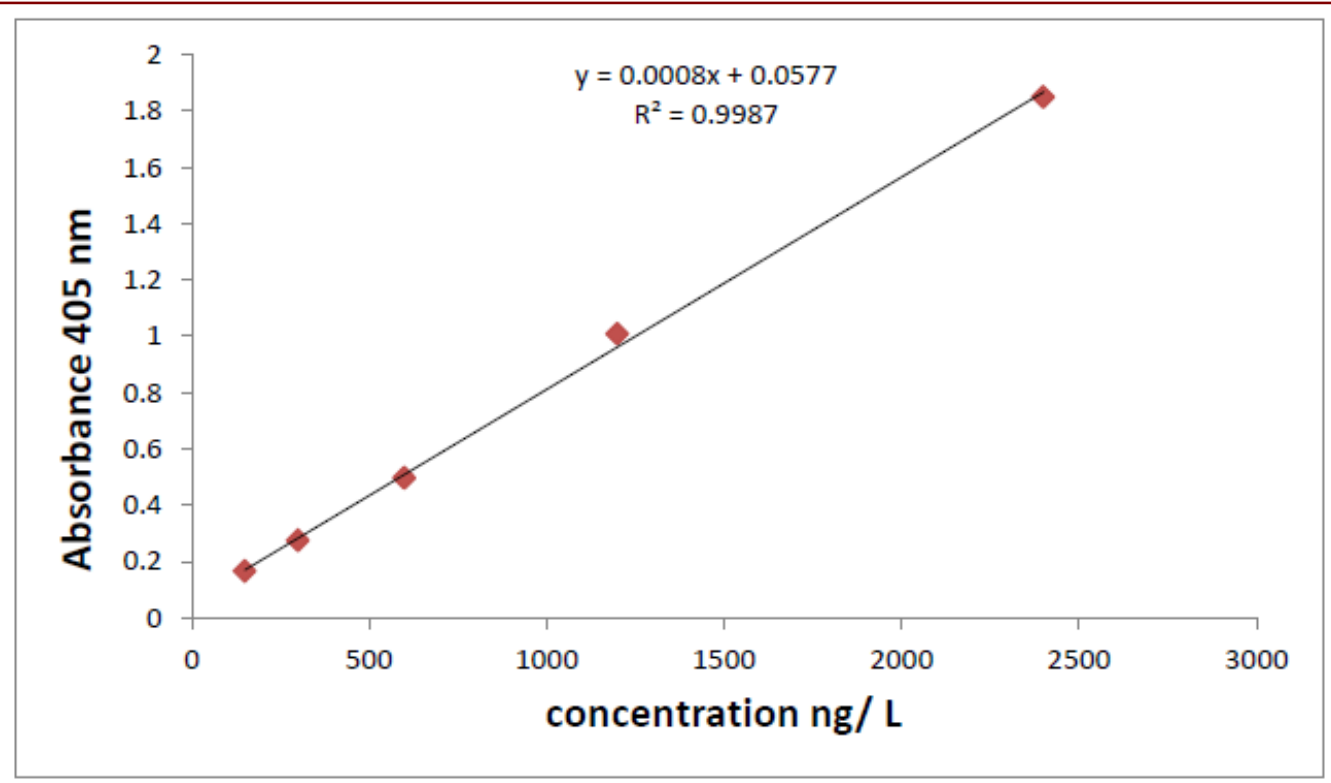

Figure.2 Standard curve of IL-33

Table.1 Mean level of IL-17A and IL-33 in sera of cutaneous leishmaniasis patients and controls

\begin{tabular}{|lc|l|l|}
\hline \multirow{2}{*}{ Serum level } & \multicolumn{2}{|l|}{ Serum level $($ Mean \pm S.E ; pg / ml) } \\
\cline { 3 - 4 } & No. & IL-17A & IL-33 \\
\hline Patients & $\mathbf{5 4}$ & $\mathbf{3 7 . 8 8} \pm \mathbf{6 . 4 1}$ & $\mathbf{2 . 7 0} \pm \mathbf{0 . 3 1}$ \\
\hline Control & $\mathbf{1 7}$ & $\mathbf{1 6 . 2 8} \pm \mathbf{2 . 0 7}$ & $\mathbf{0 . 3 4} \pm \mathbf{0 . 0 3}$ \\
\hline P- Value & $\mathbf{P} \leq \mathbf{0 . 0 1}$ & P $\leq \mathbf{0 . 0 1}$ \\
\hline
\end{tabular}

* Significant difference at the level of probability $\leq 0.01$

Table.2 Mean level of IL-17A and IL-33 in sera of cutaneous leishmainasis patients less and more than 15 years old

\begin{tabular}{|lc|c|l|}
\hline \multirow{2}{*}{ Age } & \multicolumn{2}{|l|}{ Sera level $($ Mean \pm S.E $;$ pg $/ \mathbf{m l})$} \\
\cline { 3 - 4 } & No. & IL-17A & IL-33 \\
\hline Age $<$ 15years & 36 & $\mathbf{4 0 . 5 7} \pm 4.58$ & $\mathbf{2 . 9 3 7 \pm 0 . 3 2}$ \\
\hline Age $>15$ years & 18 & $\mathbf{2 8 . 5} \pm 3.36$ & $\mathbf{1 . 8 7 3} \pm \mathbf{0 . 0 3}$ \\
\hline P-value & $\mathrm{p} \geq \mathbf{0 . 0 5}$ & $\mathrm{p} \leq 0.01$ \\
\hline
\end{tabular}

* Significant difference at the level of probability $\leq 0.01$

Table.3 Mean level of IL-17A and IL-33 in sera of males and females cutaneous Leishmaniasis patients

\begin{tabular}{|lc|l|l|}
\hline \multirow{2}{*}{ Sex } & \multicolumn{2}{|l|}{ Sera level $($ Mean \pm S.E $; \mathbf{p g} / \mathbf{m l})$} \\
\cline { 3 - 4 } & No. & IL-17A & IL-33 \\
\hline Male & $\mathbf{2 3}$ & $\mathbf{3 1 . 0} \pm \mathbf{3 . 2}$ & $\mathbf{2 . 3 4} \pm \mathbf{0 . 4 0}$ \\
\hline Female & $\mathbf{3 1}$ & $\mathbf{4 1 . 3} \pm \mathbf{4 . 1}$ & $\mathbf{2 . 9 8} \pm \mathbf{0 . 2 3}$ \\
\hline P-value & $\mathrm{p} \leq \mathbf{0 . 0 1}$ & $\mathrm{p} \geq 0.05$ \\
\hline
\end{tabular}

* No significant differences (probability $\geq 0.05$ ) 
Int.J.Curr.Res.Aca.Rev.2016; 4(5): 179-185

Table.4 Mean concentration of zinc in sera of cutaneous leishmainasis patients

\begin{tabular}{|l|c|}
\hline Sample & Concentration of zinc $($ Mean \pm S.E $; \boldsymbol{\mu m o l} /$ lit $)$ \\
\hline Patients & $5.90 \pm 0.14$ \\
\hline Control & $8.74 \pm 0.03$ \\
\hline P- Value & P $\leq 0.01$ \\
\hline
\end{tabular}

Table.5 Mean concentration of iron in sera of cutaneous leishmainasis patients

\begin{tabular}{|l|l|}
\hline Sample & Concentration of iron $($ Mean \pm S.E $; \boldsymbol{\mu m o l} /$ lit $)$ \\
\hline Patients & $\mathbf{1 4 . 2 2} \pm 0.36$ \\
\hline Control & $22.12 \pm 0.73$ \\
\hline P-Value & $\mathbf{P} \geq 0.05$ \\
\hline
\end{tabular}

Table.5 Comparison of adenosine deaminase activity in patients with cutaneous leishmaniasis and control group

\begin{tabular}{|l|l|}
\hline Sample & Concentration of iron $($ Mean \pm S.E $; \mu \mathrm{mol} /$ lit $)$ \\
\hline Patients & $\mathbf{1 4 . 2 2} \pm 0.36$ \\
\hline Control & $\mathbf{2 2 . 1 2} \pm 0.73$ \\
\hline P-Value & $P \geq 0.05$ \\
\hline
\end{tabular}

Table.6 Comparison of adenosine deaminase activity in patients with cutaneous leishmaniasis and control group

\begin{tabular}{|l|l|}
\hline Sample & adenosine deaminase activity (Mean \pm S.E; unit/lit) \\
\hline Patients & $\mathbf{1 2 . 6 3} \pm 0.64$ \\
\hline Control & $5.66 \pm 0.21$ \\
\hline P-Value & $\mathbf{P} \leq 0.01$ \\
\hline
\end{tabular}

In this investigation, we assessed the levels of selected cytokines in the sera of patients had been positively diagnosed with cutaneous leishmaniasis. Cytokines have a central role in the immune response, presenting local and systemic effects (Bogaart et al., 2014). It seems that Th1/ Th2 cytokine balance may be involved in the resistance or susceptibility to leishmaniasis infection. In this respect, Th1 cytokines confer resistance that is dependent upon expression of IL-12, IFN- $\gamma$ and TNF- $\alpha$ with production of nitric oxide (NO) (Mutiso et al., 2013). In contrast, Th2 cytokines confer to susceptibility that is associated with a regulatory response with the concomitant production of IL-10 as well as expression of Th2 cytokines such as IL-4 and TGF- $\beta$ (Lopez et al., 2014).
Therefore, each cytokine influencing this balance can affect the outcome of the infection with leishmaniasis. One of these cytokines is IL-17A, representing an important link between the innate and adaptive immune response. Interleukin- 17A (IL-17A) is known to play a role in inflammation and has multiple effects, acting both in the induction of proinflammatory cytokines and in the recruitment and activation of leukocytes. Thus, it seems that IL-17 can affect the induction of Th1 immunity which is necessary for controlling leishmaniasis and it is also seems to play an important role in the pathogenesis of the disease (Olivia et al., 2009; Espir et al., 2014). Our data point to higher levels of IL-17 in the sera of patients with cutaneous leishmaniasis is in 
agreement with Bacellar et al (2009) who observe that IL-17 is produced during infection with $L$. braziliensis which is associated with neutrophils and tissue destruction. It is also observed that lymphocytes obtained from patients with cutaneous leishmaniasis yielded higher concentrations of IL-17 compared to lymphocytes obtained from uninfected individuals (Bacellar et al., 2009).

Interleukin -33 (IL-33) appears to be a crucial cytokine for Th2 mediated host defense and play a central role in controlling immune response in barrier tissues such as skin and intestine. It is able to activate cells of both the innate and adaptive immune system and depending on the disease can either promote the resolution of inflammation or drive disease pathology $(\mathrm{Li}$ et al., 2014). In addition to promoting Th2 responses, IL-33 exhibits proinflammatory potential by inducing the production of a number of inflammatory mediators (Huynh et al., 2002). Consistent with other observations, our report of significantly increased level of IL-33 in the serum of patients infected with cutaneous leishmaniasis, represents a promising new therapeutic strategy for treating or preventing various inflammatory disorders. However, many questions regarding the fundamental biology of IL-33 remain to be solved including its nuclear effects and processing and release of IL-33 from cells.

The present study shows that the patients with cutaneous leishmaniasis had low serum zinc and iron levels than those of healthy subjects, due to the leishmaniasis and the decreased zinc and iron bioavailability in their diet. The decreased levels of zinc and iron in sera of patients could also be a useful marker for immune dysfunction in leishmaniasis. On the other hand significant increase in the activity of adenosine deaminase in sera of patients with cutaneous leishmaniasis as compared to healthy control could be a useful marker for cellular immune response. Adenosine deaminase play an important role in the growth and differentiation of T-lymphocytes which involved in the secretion of interleukin.

\section{References}

Al- Warid, H.S.J. 2007. Study for the growth rate, viability and morphological changes of Leishmania tropica in different culture media. Journal of AlNahrain University, 10: 136-135.

Awasthi, A., Mathur, R.K., Saha, B. 2004. Immune response to Leishmania infection. Indian J. Med. Res., 119: 238258.

Bacallar, O., Faria, D., Nascimento, M., Cardoso, T.M., Gollob, K.J., Dutra, W.O., Scott, P., Carvalho, E.M. 2009. "Interleukin 17production among patients with American cutaneous leishmaniasis," J. Infect. Dis., 200: 7578.

Bogaart, E.V., Talha, A.A., Straetemans, M., Mens, P.F., Adams, E.R., Grobusch, M.P., Nour, B.Y., Schallig, H.D.F. 2014. Cytokine profiles amongst Sudanese patients with visceral leishmaniasis and malaria co-infections. Bio. Med. Cent. Immunol., 15: 1-10.

Carter, C.R., Whitcomb, J.P., Campbell, J.A., Mukbel, R.M., McDowell, M.A. 2009. Complement Receptor 3 Deficiency Influences Lesion Progression during Leishmania major Infection in BALB/c Mice. Infect. Immunity, 77: 5668-5675.

Espir, T.T., Figueira, L.de. P., Naiff, M., Costa, A.G., Ramalho-Ortiogao, M., Malheiro, A., Franco, A.M.R. 2014. The Role of Inflammatory, AntiInflammatory, andRegulatory Cytokines in Patients Infected with Cutaneous Leishmaniasis in Amazonas State, Brazil. J. Immunol. Res., 1-10. 
Garnier, T., Croft, S. 2002. Topical treatment for cutaneous leishmaniasis. Curr. Opi. Investigational Drugs, 3: 1472-4472.

Giusti, G. 1980. Adenosine deaminase. In: Methods of Enzymatic Analysis $2^{\wedge}$ nd ed. Bergmeyer, H. V., 2: 1092-1099. Verlage. Chemio. International, Florida.

Gonzalez, U., Pinart, M., Rengifo-Pardo, M., Macaya, A., Alvar, J., Tweed, J.A. 2009. Interventions for American cutaneous and mucocutaneous leishmaniasis (Review). The Cochrane Collaboration, 2: 1-171.

Huynh, M.L., Fadok, V.A., Henson, P.M. 2002. Cytokine and cytokine receptors. J. Clin. Invest., 41-50.

Kirgbright, G.F. 1980. Atomic absorption spectroscopy, Elemental analysis of biological materials. Vienna Technical Report Series. Int. Atomic Agency, 197: 141-165.

Li, C., Li, H., Jiang, Z., Zhang, T., Wang, Y., Li, Z., Wu, Y., Ji, S., Xiao, S., Ryffel, B., Radek, K.A., Xia, Z., Lai, Y. 2014. Interleukin-33 Increases Antibacterial Defense by Activation of Inducible Nitric Oxide Synthase in Skin. PLoS Pathog., 10(2): 1-14.

Liu, D., Uzonna, J.E. 2012. The early interaction of Leishmania with macrophages and dendritic cells and its influence on the host immune response. Frontiers in Cellular and Infection Microbiol., 2: 1-8.

Lopez, K.S., Dinges, S., Griewank, K., Iwakura, Y., Udey, M.C., von, S.E. 2009. IL-17 promotes progression of cutaneous leishmaniasis in susceptible mice. J. Immunol., 182(5): 3039-3046.
Mutiso, J.M., Macharia, J.C., Gicheru, M. M., Ozwara, H. 2013. Immunology of leishmaniasis. J. Sci. Parasitol., 14(2): $51-61$.

Olivia, B., Daniela, F., Marcia, N., Thiago, M.C., Kenneth, J.G., Walderez, O., Dutra, P.C., Edgar, M.C. 2009. Interleukin 17 Production among Patients with American Cutaneous Leishmaniasis. J. Infect. Dis., 200: 758.

Rahi, A.A. 2013. Cutaneous Leishmaniasis in Iraq: A clinicoepidemiological descriptive study. Scholars J. Appl. Med. Sci., 6: 1021-1025.

Rahi, A.A., Hraiga, B.A., Hassoni, J.J. 2014. Some Epidemiological Aspects of Cutaneous Leishmaniasis in Kut city, Iraq. Scholars J. Appl. Med. Sci., 2: 451-455.

Rassam, M., Al-Mudhaffar, S., Chance, M. 1979. Isoenzym characterization of Leishmania species from Iraq. Ann. Trop. Med. Parasite., 73: 527-534.

Sehgal, R., Goyal, K., Kanwar, R., Sehgal, A., Kanwar, J.R. 2012. Immunology of leishmaniasis and future prospective of vaccines. Sharma, U., Sood, A., Arora, S., Kanga, A., Mahajan, V., Negi, A., Sharma, A. 2008. Characteristics of Leishmania spp. Isolated from a mixed focus of cutaneous and visceral Leishmaniasis in Himachal Pradesh (India). The Internet of Third World Medicine; 7: 1-7.

Sharma, U., Singh, S. 2009. Immunobiology of leishmaniasis. Indian. J. Exper. Biol., 47: 412-423.

\section{How to cite this article:}

Husain F. Hassan, Sahla Kh. Abbas and Dunya S. Shakoor. 2016. Evaluation of Interleukin IL17A, IL-33, and Serum Levels of Zinc, Iron and Adenosine Deaminase in Cutaneous Leishmaniasis in Kirkuk, Iraq. Int.J.Curr.Res.Aca.Rev.4(5): 179-185.

doi: http://dx.doi.org/10.20546/ijcrar.2016.405.018 